\title{
Quaderni
}

QUADERN I Communication, technologies, pouvoir

\section{De l'incitatif économique à la machine de gestion : le cas des établissements de santé}

Jean-Claude Moisdon

\section{(2) OpenEdition}

Édition électronique

URL : http://journals.openedition.org/quaderni/741

DOI : 10.4000/quaderni.741

ISSN : 2105-2956

Éditeur

Les éditions de la Maison des sciences de l'Homme

Édition imprimée

Date de publication : 5 octobre 2013

Pagination : 39-54

Référence électronique

Jean-Claude Moisdon, « De l'incitatif économique à la machine de gestion : le cas des établissements de santé », Quaderni [En ligne], 82 | Automne 2013, mis en ligne le 05 octobre 2015, consulté le 01 mai 2019. URL : http://journals.openedition.org/quaderni/741 ; DOI : 10.4000/quaderni.741 


\section{$D$ ossier}

De l'incitatif économique à la machine de gestion : le cas des

\section{établissements}

de santé

Jean-Claude Moisdon

Directeur de recherche émérite à l'École de Mines de Paris
Gouverner par les instruments tel est le titre de l'ouvrage de Pierre Lascoumes et Patrick Le Gales ${ }^{1}$, paru en 2004, manifestant un intérêt croissant de la recherche pour saisir le sens de l'action publique non dans les objectifs déclarés, intentions, discours du politique, mais dans les dispositifs techniques qu'elle met en œuvre. Ces supports de la pensée, cartographies, statistiques, ratios, modèles, etc., n'avaient guère fait l'objet d'analyses savantes, étant considérés comme des corollaires secondaires des intentions du législateur. Or les auteurs montrent qu'il peut être heuristique d'y prêter attention, non seulement parce qu'ils constituent des traceurs d'une politique, condensant les controverses qui la façonnent, mais aussi parce qu'ils manifestent, lors de leur insertion dans le tissu social une relative autonomie, provoquant des effets collatéraux et des déviations de trajectoire inattendues.

Cette approche est voisine de celle qu'avaient développée auparavant un certain nombre de chercheurs sur un autre champ, celui de la gestion des organisations. Prenant acte du fait que, depuis le milieu du dix-neuvième siècle un outillage gestionnaire avait progressivement émergé de l'édifice comptable pour proliférer dans les secteurs les plus divers, de l'industrie à la culture en passant par la santé, ils avaient eux-aussi étudié les divers effets d'une telle expansion des artefacts de la gouvernance d'entreprise $^{2}$. C'est dans cette lignée de recherche en gestion que cette contribution s'inscrit, mais elle se trouve étroitement connectée à celle de l'évaluation des politiques publique, par l'analyse qu'elle propose d'une instrumentation particulière, la tarification à 
l'activité des établissements de santé (T2A), à la fois instrument de régulation du système hospitalier et instrumentation de gestion des hôpitaux eux-mêmes.

Nous présenterons tout d'abord ce nouveau mode de financement, emblématique du NPM (New Public Management), censé créer davantage d'équité et de transparence dans l'allocation des ressources aux hôpitaux, mais aussi adresser à ces derniers un signal économique les conduisant à un surcroît d'efficience par rapport à une situation caractérisée, dans l'esprit du régulateur, par de nombreuses zones de sousproductivité.

Nous ferons alors état des effets de cette instrumentation, en tout cas ce que l'on en connaît à l'heure actuelle. Par rapport aux objectifs affichés d'équité, d'efficience, et de transparence, le bilan est tempéré, pour des raisons que nous avancerons, et qui tiennent aussi bien à l'outil lui-même qu'aux caractéristiques organisationnelles de l'activité hospitalière. Si les dégâts collatéraux redoutés par de nombreux observateurs quant à la qualité et la sécurité des soins, susceptibles de souffrir dans un système de marché ou de pseudo-marché, ne sont pas avérés, il manque manifestement des analyses approfondies sur la question.

D’une façon générale, les modalités concrètes de la régulation actuelle ne profitent pas assez à nos yeux d'un effet majeur de l'instrumentation de gestion, à savoir les effets d'apprentissage. Contrairement à la philosophie d'action qui est à la base de la T2A, à savoir l'orientation des comportements par un régulateur éloigné, et qui donc n'a pas à se soucier des fonctionnements concrets, qu'il juge trop complexes, l'usage d'instruments imparfaits et lacunaires ne peut supprimer la discussion et la communication ; il les amplifie, en les régénérant. C'est à ce prix que savoir et action peuvent se construire conjointement. Gouverner par les instruments signifie aussi gouverner les instruments.

\section{La tarification à l'activité}

La T2A est née en 2004 pour les hôpitaux publics ou participants au service public et en 2005 pour les cliniques privées, mais elle a été préparée par une évolution qui a commencé au milieu des années soixante-dix. Auparavant, le système hospitalier français avait connu une forte croissance, marquée par une régulation permissive et une absence notable de philosophie et d'instrumentation gestionnaires. L'outil princeps est le budget, calculé à partir du nombre de journées et du prix des journées, fixé par les services déconcentrés de l'État, système considéré comme inflationniste mais restant durablement en place de la Seconde Guerre mondiale jusqu'au début des années quatrevingt.

La crise des déficits publics a changé la donne. Un rationnement tente de s'installer à partir du milieu des années soixante-dix et en 1983 est instauré ce qui est considéré par beaucoup comme une chape de plomb : la dotation globale, forfait annuel accordé à chaque hôpital public (les établissements privés continuant à être rémunérés à la journée ou à l'acte). Ce système se révèle vite insatisfaisant aux yeux de la plupart des acteurs : les hôpitaux estiment 
qu'ils ne peuvent pas se développer, tout en réussissant à desserrer continuellement la contrainte, aussi bien grâce aux jeux possibles permis par l'édifice comptable que par le lobbying auprès du politique. Calculées à partir des bases budgétaires initiales, les dotations enregistrent les rentes de situation ou au contraire des situations défavorables laissées par la régulation opaque précédente. De toute façon, peu de progrès sont enregistrés quant à la visibilité du système. De nombreuses questions (le système est-il efficient? Est-il équitable? Offre-t-il des prestations assurant la qualité des soins et la sécurité des patients ?) restent sans réponse autre qu'idéologiques et la plupart du temps négatives.

Mais à la même époque, sous l'impulsion des cadres de l'Administration Centrale, essentiellement la Direction des Hôpitaux, un changement se dessine quant à l'instrumentation de contrôle de l'activité hospitalière : à partir de 1983 se développe le projet PMSI (Projet puis Programme de Médicalisation de l'Information), directement inspiré d'expériences nordaméricaines, consistant à mettre sur pied une information de signification à la fois médicale et économique : les séjours hospitaliers sont classés dans des catégories (les GHM : Groupes Homogènes de Malades), qui sont en fait des regroupements de pathologies ou d'actes invasifs (par exemple " gastroentérologies " ou « interventions majeures sur l'intestin grêle) et qui sont censés consommer des niveaux de ressources voisins. Au début du projet on a affaire à environ 600 groupes, s'inspirant directement de l'expérience américaine, fondée sur des modèles statistiques de segmentation appliqués à des dizaines de milliers de séjours.

Par ailleurs, à partir de 1992, un dispositif, appelé Étude Nationale de Coûts (ENC) permet d'estimer chaque année sur un échantillon d'établissements, et grâce à une comptabilité analytique standardisée, des coûts complets par séjour, et donc des coûts moyens par GHM.

Plusieurs remarques doivent être faites à ce niveau :

- L'enrichissement considérable de l'information: on passe grosso modo d'un hôpital décrit par un nombre global de séjours, indifférenciés médicalement, à un hôpital décrit par un « case mix », fait de 600 nombre de séjours à connotation médicale. Gardons cependant à l'esprit que la classification internationale des maladies comporte plus de 15000 pathologies et que l'ENC conduit à de fortes variabilités de coûts dans un même GHM. Par ailleurs ce dispositif n'a pour le moment été concrétisé que pour les soins dits aigus (hors rééducation et psychiatrie).

- Corrélativement l'ampleur et la multiplicité des savoirs mobilisés dans cette opération : médicaux, statistiques, informatiques, comptables, juridiques, mais aussi économiques. Le processus d'acculturation des cadres de l'administration de la santé aux théories économiques provenant du monde anglo-saxon a été décrit par ailleurs ${ }^{3}$. On peut considérer que le système américain de financement des hôpitaux, issu de la catégorisation des séjours, s'inspire directement des travaux des chercheurs qui, par des modélisations souvent sophistiquées, ont tenté de surmonter les défis qu'opposaient à l'équilibre walrassien 
les problèmes d'asymétrie d'information ou d'externalités posés par le produit santé. Il en sera de même plus tard pour la T2A française (et pour les dispositifs analogues dans tous les pays développés).

- Le projet a été particulièrement long et on verra d'ailleurs qu'il n'est pas encore achevé. Démarré en 1983, il a conduit à une tarification en 2004. Ce ne sont pas seulement les très nombreux problèmes techniques rencontrés qui sont en cause, mais également une valse hésitation durable du politique quant à l'usage d'un tel outillage : simple instrument de contrôle et de pilotage pour les managements hospitaliers ? Ou pour les tutelles locales des établissements (DDASS et DRASS, ARH Agences Régionales de l'Hospitalisation - à partir de 1996) ? Tarification à l'américaine ? L'État, longtemps, a « avancé sur des œufs », le PMSI notamment ayant reçu une avalanche de critiques de la part du monde des hôpitaux -corps médical en tête- dès qu'il a pointé le bout du nez. D'ailleurs, entre les premiers résultats de l'ENC en 1995 et la tarification en 2004, le dispositif n'a préconisé que l'usage d'un indicateur, censé être utilisé par les ARH : comme en 1992 une circulaire impose à tous les établissements de fournir pour chaque patient l'information de base (diagnostics, acte invasif, âge etc.) permettant de le classer dans un GHM ; l'État dispose pour chaque hôpital, de son case-mix, transmis aux ARH. Il suffit alors d'appliquer à ce case-mix les coûts moyens obtenus par l'ENC pour disposer d'un budget théorique (correspondant à une consommation moyenne de ressources alignée sur celle de l'échantillon de l'ENC), et ce pour chaque établissement. Le rapport entre le budget réel de l'établissement et ce budget théorique fournit donc un indicateur d'efficience pouvant être mobilisé - à volonté dans les négociations budgétaires.

Si les valeurs de cet indicateur révélèrent des écarts considérables (sur le territoire national, un établissement, à l'aune de l'instrument, pouvait consommer trois fois plus de ressources qu'un autre pour une même activité), elles n'ont ensuite que peu évolué, montrant une utilisation variable, mais toujours parcimonieuse, par les ARH.

C'est cette constatation, doublée d'un phénomène d'isomorphisme mimétique (pour emprunter un terme aux institutionnalistes), la plupart des pays développés développant un système de financement par tarification s'inspirant de l'expérience américaine, qui a conduit le centre à reprendre la main et à instaurer la T2A en 2004.

Cette dernière, dont on vient d'expliciter l'origine et les grandes lignes, mérite quelques commentaires : il est clair tout d'abord qu'elle ne vise, dans ses principes, que l'efficience, c'est-à-dire in fine le niveau des dépenses. Elle rémunère un séjour suivant un forfait, ne dépendant que du GHM dans lequel est classé le séjour. Par rapport à d'autres modes de financement, tel le prix de journée, ou encore le paiement des actes, elle provoque un changement considérable : on n'a plus intérêt à laisser les séjours s'allonger ou à multiplier les actes. Ce qui est espéré, c'est que les hôpitaux, pris dans cet étau, repèrent et pourchassent leurs zones de sous-productivité, profitant du signal économique offert par la T2A, pour conserver 
ou obtenir l'équilibre économique.

Les objectifs corollaires sont d'une part la possibilité de financer le progrès technique, de plus en plus difficile à préserver dans le contexte des déficits publics, et d'améliorer l'équité dans l'allocation des ressources aux établissements (et donc dans l'accès à ses ressources), ce que ne permettait pas le système de dotation globale.

Le dispositif en revanche, en tout cas dans ses débuts d'application, ne dit rien sur la qualité des soins et la sécurité des patients (il convient de signaler que rien n'était dit non plus sur ces sujets dans les régulations précédentes). De même, il ne prend pas en charge, là aussi dans ses grands principes, certaines missions dites de service public, telles la recherche médicale ou encore l'accueil des populations précaires, dont certains établissements ont davantage la charge (en occurrence les établissements publics par rapport au secteur privé). En conséquence, comme on peut l'imaginer, la T2A n'a pas été et n'est toujours pas sans susciter de très nombreuses critiques ${ }^{4}$, invoquant le thème de la mise sur le marché de la santé et de privatisation, ou d'inquiétudes, du reste légitimes, quant à la génération possible de comportements opportunistes liés à ce type d'incitatif économique : sélection des patients à l'entrée, surcodage, « saucissonnage » des séjours, etc.

Dans les faits qu'observe-t-on après huit années d'instauration de ce mode de financement?

\section{Effets observables et opacités}

On ne peut pas dire que l'on dispose à l'heure actuelle d'une évaluation en bonne et due forme de la T2A. Les impacts sur les comportements des producteurs de soins et sur le fonctionnement des hôpitaux sont particulièrement peu informés, alors que l'objectif affiché était une amélioration de l'efficience des processus de soins, et ce par le fait de l'incitatif économique et de la richesse informationnelle qu'il contient. Les observations dont on dispose toutefois à ce titre ${ }^{5}$ montrent que peu de réorganisations internes aux établissements se sont mises en place. On note des efforts du côté des achats, de la soustraitance ou encore de la facturation, mais elles se situent dans la poursuite des tendances précédentes et manifestement les acteurs ne se sont guère saisis de la déclinaison du signal en 2400 items (le nombre de GHM actuel).

En revanche, des statistiques existent en grand nombre sur l'activité (nombre de séjours, par type de séjours, pondérés par des poids par GHM etc.) et elles montrent une croissance de la production hospitalière, surtout pour le secteur public, au delà de ce que supposerait l'évolution de la démographie. Cette augmentation est-elle toujours pertinente, au sens de sa justification médicale ? Cette question n'a pour le moment pas de réponse. Par ailleurs l'observation de l'évolution des effectifs de soignants montre que ces derniers augmentent moins vite que l'activité, en tout cas pour les paramédicaux, ce qui relèverait davantage d'une intensification du travail que d'efforts de réorganisation, d'après ce qui précède.

Sur les craintes concernant la qualité des soins et la sécurité des patients, des statistiques existent également, mais peu concluantes pour le moment, 
ne montrant pas d'effets notables. Par exemple une étude de la mortalité post-hospitalière de quelques pathologies lourdes ${ }^{6}$ ne marque pas de rupture depuis 2005 ; l'analyse des taux de réadmission fournit des résultats ambigus, variables selon les GHM ; les files d'attente pour certains traitements (chimiothérapies) ont plutôt tendance à diminuer ${ }^{7}$. Des indicateurs de qualité ont été mis en place par la Haute Autorité de Santé et la DGOS, mais d'une part leur développement est trop récent pour évaluer une influence de la T2A, d'autre part il s'agit d'indicateurs de processus, et non de résultats. Quant aux effets d'opportunisme, en dehors de l'éventuelle induction de la demande évoquée ci-dessus, on a du mal à repérer des stratégies de sélection à l'entrée des hôpitaux publics, en tout cas dans les données d'activité.

Comment interpréter ces faits?

Provisoirement nous aurions tendance à mettre en avant trois types de causalité :

- La caractéristique essentielle de l'hôpital comme organisation professionnelle ${ }^{8}$, avec le thème très connu des « motivations intrinsèques " des soignants, médecins et paramédicaux. Cette caractéristique ne va pas évidemment s'évanouir par le fait de l'irruption de la T2A, vis-à-vis de laquelle beaucoup, au mieux, manifestent une solide indifférence mais d'un autre côté, celle-ci semble davantage acceptée, dans ses principes généraux en tout cas, que les systèmes précédents.

- La complexité et l'incertitude du signal économique et de ses traductions instrumentales pour la gestion interne. C'est là que l'on quitte fatalement le monde parfait des théorèmes économiques pour entrer dans celui imparfait du monde gestionnaire, fait à la fois de science, de technique, d'arbitraire, et d'à peu près. Sans entrer dans une énumération fastidieuse et obligatoirement très longue, donnons quelques exemples concernant la T2A : comme on l'a dit il y a, à l'heure actuelle, 2400 GHM, ce qui constitue d'emblée un dispositif d'une certaine lourdeur, mais encore très synthétique par rapport aux classifications internationales et surtout par rapport à ce qui peut constituer une trajectoire singulière de patient : l'âge, le sexe, les complications, l'histoire individuelle, etc., tout ce qui fait que l'adage du malade unique chère aux soignants a une part de vérité, malgré des efforts de protocolisation incontestables. On a là la convention gestionnaire la plus fréquente, mais aussi la plus redoutable : la convention d'assimilation, qui fait que pour éviter des systèmes ingouvernables (des millions de GHM), on confond une unité (de production ou de ressource) avec une autre, que l'on mélange des objets que l'on dit identiques et qui ne le sont pas, sans malheureusement que l'on puisse évaluer l'erreur que l'on commet en opérant de la sorte. Autre exemple concernant les coûts de référence fournis par l'ENC : comme certains analystes lucides des pratiques comptables l'ont démontré', un coût n'existe pas. Car il y a de multiples façons de le calculer, notamment dans le cadre d'une comptabilité analytique qui impose que l'on rattache à une unité de production (un séjour) l'ensemble des dépenses des unités qui participent de près ou de loin à sa production. Cela ne peut se faire (songeons aux services administratifs) que par l'intermédiaire 
de clefs de répartition qui sont de l'ordre du « choix raisonnable ». À ces ventilations dans l'espace, il faut ajouter des ventilations dans le temps des investissements, qui obéissent également à des règles conventionnelles, qui peuvent donc varier. Dernier exemple : comment intégrer au dispositif les missions de service public, qui correspondent à l'usage de ressources qui ne sont pas affectables à des patients, comme l'enseignement et la recherche ? Problème difficile, qui a donné lieu à de multiples approches, d'autant qu'isoler rigoureusement cette activité de celle liée aux soins est impossible. Faute de mieux le choix a été fait de conserver une part des ressources allouées aux hôpitaux sous forme de dotation forfaitaire, conventionnellement calibrée à un niveau global, mais répartie entre les établissements selon un modèle complexe dépendant d' indicateurs censés représenter l'effort des uns et des autres dans ce domaine (nombre de publications scientifiques, d'essais cliniques, d'étudiants etc.). Il faut ajouter que l'État a par ailleurs surchargé le dispositif d'objectifs autres que ceux initialement affichés, en déformant les tarifs issus des coûts de l'ENC pour favoriser un certain nombre de priorités des santé publique, dans des proportions là aussi largement conventionnelles, ou encore pour rapprocher les tarifs du public ou du privé.

$\mathrm{Au}$ total le système s'est considérablement complexifié non seulement au niveau technique (2400 GHM, des listes de milliers de diagnostics et d'actes, des centaines de règles plus ou moins arbitraires aboutissant aux tarifs etc.), par un processus de perfectionnement d'un outil qui ne sera jamais parfait, mais aussi au niveau de sa signification même : on a affaire à un hybride entre marché et planification, à un mélange entre des forfaits et des incitatifs divers, où les coûts, donc l'efficience, ne constituent plus la visée unique. À l'heure actuelle, il est quasiment impossible à un acteur local, manager ou professionnel de soin, de comparer leurs niveaux de ressources aux tarifs, d'autant que ces derniers évoluent d'une année sur l'autre, le modèle étant régulièrement modifié.

- Troisième cause de l'absence des faibles changements constatés en interne : les caractéristiques organisationnelles de l'hôpital. L'incitatif économique conduit théoriquement, on l'a dit, les établissements à réformer leurs processus de soins. C'est d'une certaine façon les amener à considérer leur propre institution comme un système de production, où il s'agirait de structurer des gammes de production (les trajectoires de patients) et d'ordonnancer ces gammes sur les différents points de traitement (service clinique, bloc, imagerie etc.). C'est là un modèle d'action auquel les acteurs hospitaliers ne sont pas du tout habitués, ni formés, ni soutenus en interne ; il n'existe ni service méthode, ni service ordonnancement. Or l'hôpital vu comme système de production est remarquablement complexe. Sans là aussi entrer dans les détails, soulignons simplement que les trajectoires de patients sont conçues indépendamment les unes des autres (par les médecins) tout en étant dans les faits interdépendantes, de même qu'elles sont dépendantes d'autres processus généraux, comme ceux qui concernent l'hôtellerie, ou encore le circuit du médicament. Elles font intervenir, de façon variable un grand nombre d'acteurs, aux logiques professionnelles différentes, et sont aléatoires, même si la 
médecine protocolée tend à se généraliser ${ }^{10}$. Un tel « atelier » défie a priori toute tentative de rationalisation instrumentale.

Il y a là une contradiction entre un incitatif économique, en quelque sorte vertical, qui pèse sur un acteur, souvent à présent un médecin responsable de pôle ${ }^{11}$, ayant officiellement autorité sur un périmètre particulier de l'hôpital, engagé dans une logique contractuelle avec la direction, et une organisation plutôt horizontale, faite d'un enchevêtrement de multiples processus, débordant le périmètre en question et mis en œuvre par des acteurs largement autonomes. Une réorganisation dans ce contexte constitue toujours une aventure difficile, coûteuse en investigations et négociations de toutes sortes, jamais définitivement acquise, aboutissant fréquemment à l'épuisement de celui ou de ceux qui l'entreprennent, même si des expériences menées ici ou là, se sont révélées positives et si l'État, conscient du problème, a mis sur pied une expertise sous la forme d'une agence (ANAP : Agence Nationale d'Appui à la Performance des établissements de santé et médicosociaux).

Dans l'immédiat, les constatations précédentes expliquent à nos yeux pourquoi, au lieu de se livrer à de pénibles efforts de réduction des coûts, les hospitaliers ont préféré explorer les possibilités d'augmentation des recettes, en se laissant guider davantage par les opportunités du moment que par un incitatif riche en informations médicales, mais ininterprétable et trop exigeant sur le plan du management.

C'est là sans doute que l'instrument de gestion, un dégradé de théories économiques, comme on vient de le voir, se transforme finalement en mécanisme de gestion : en effet, le développement de l'activité hospitalière se heurte de toute façon à une contrainte extérieure à présent impérative : l'ONDAM (Objectif National des Dépenses d'Assurance Maladie), voté chaque année par le parlement ainsi que ses déclinaisons, notamment la sous-enveloppe allouée à l'hospitalisation. Celle-ci n'évolue plus d'une année sur l'autre qu'au mieux de $3 \%$, et ne peut supporter à la fois l'évolution des coûts de production et l'activité. Comme l'État, dans une optique de régulation prix/volume, intègre l'évolution de l'activité dans la fixation des tarifs, ceux-ci ne peuvent que s'éloigner des coûts, et aggraver les écarts existants, rendant le signal économique de moins en moins clair.

D'une certaine façon, la philosophie de la boîte noire ${ }^{12}$, qui préside à ce dispositif, inspiré par les approches micro-économiques récentes, semble s'être retournée contre le régulateur : ne pas aller y voir et adresser aux acteurs un signal économique suppose que ces derniers pourront s'orienter avec le signal comme guide ; mais ce n'est pas le cas. La boîte est noire pour eux aussi.

Finalement, qu'est ce que la T2A ?

Techniquement il ne s'agit plus de l'application d'un théorèmeéconomique, mais d'uninstrument imposant, sinon pharaonique, qui répartit une enveloppe globale nationale sur 3000 entités environ, selon des clefs de ventilation ayant des rapports plus ou moins lointains avec des coûts de production de référence, façonnées par de 
multiples conventions de calcul et intégrant par ailleurs les priorités du moment.

$\mathrm{Au}$ niveau des résultats, il convient d'être très prudent, mais, en attendant des évaluations plus consistantes que celles qui existent actuellement, il semble bien que le premier objectif affiché, à savoir l'efficience, ne soit pas à la hauteur des attentes, pour les raisons que l'on vient d'évoquer. De même, on ne peut pas dire que la transparence du modèle de financement ait été vraiment améliorée. En revanche, on peut penser que l'allocation des ressources est plus équitable qu'auparavant, les écarts entre établissements ayant été fortement réduits (à l'aune de l'instrument lui-même cela dit, ce qui ne clôt pas la question, compte tenu de la façon dont on vient de décrire ce dernier). Par ailleurs, la T2A a clairement libéré des capacités stratégiques du côté des établissements, surtout ceux du secteur public, qui étaient manifestement bridées par la dotation globale.

Si pour le moment, les inquiétudes en matière de qualité, de sécurité, d'effets pervers, ne se sont pas concrétisées, cela pourrait signifier que les pratiques professionnelles auraient plutôt bien résisté. Mais là aussi il parâ̂t difficile de se contenter à l'avenir des quelques études qui existent.

On voit combien il est difficile d'évaluer la mise en place d'un tel dispositif. Les déclarations péremptoires en matière d'échec ou au contraire de progrès spectaculaire, ou encore de destruction du service public livré au marché et à la privatisation, ne paraissent pas à la hauteur des multiples facettes de la réforme et des ambigüités de ses résultats.

De nombreuses critiques s'adressent à l'heure actuelle à l'opacité du dispositif et de ses effets, si bien qu'une réflexion d'ensemble a été entreprise par le nouveau gouvernement, sachant qu'un retour en arrière (suppression de la T2A) semble exclu. Par ailleurs, le peu de certitudes disponibles quant à l'évolution de la qualité des soins a conduit à expérimenter un rééquilibrage de l'incitatif, par intégration des indicateurs disponibles.

Cette opération de réfection d'ensemble s'avère complexe, et il n'est pas certain que le régulateur dispose de tous les éléments de connaissance pour ce faire, car on peut se demander si le développement de ce projet n'a pas insuffisamment profité d'un autre type d'effets, plus impalpables que ceux que l'on vient d'évoquer, mais structurants à la fois pour le cas étudié ici, et plus généralement pour les recherches actuelles sur les organisations ${ }^{13}$ : les effets d'apprentissage liés à l'instrumentation.

\section{Apprentissages, savoirs actifs, savoirs manquants}

Trois types d'effets de connaissance sont à mettre au crédit des outils de gestion ${ }^{14}$ :

- La fonction traditionnellement dévolue à l'instrument de gestion est désignée par le terme d'aide à la décision, mais, compte tenu de la nature de cet objet, il est préférable d'utiliser l'expression « aide à l'exploration du nouveau » Pour l'essentiel il s'agit toujours de modéliser plusieurs interactions entre variables 
que l'esprit ordinaire d'un décideur ou ici d'un régulateur ne saurait faire jouer ensemble. Cette instrumentation, relativement nouvelle car née avec la grande entreprise au milieu du dix-neuvième siècle, proliférant ensuite et s'attaquant aux secteurs les plus divers et souvent inattendus (culture, éducation..), offre donc une sorte de prothèse intellectuelle à ce qui caractérise toute organisation à partir d'une taille du reste modeste, la rationalité limitée. On saisit ici ce qui fait la séduction d'une approche qui a conduit à une sorte de rêve, culminant dans les années cinquante, pensant l'action collective par sa mathématisation. Sans entrer ici dans les multiples considérations qui ont conduit experts et décideurs à retomber sur terre, nous mettons pour notre part l'accent sur une donnée technique générale, qu'il s'agisse du PMSI, de la T2A ou d'un quelconque outil de gestion, c'est-à-dire leur fragilité en tant que synthèse inaboutie, discutable, faite de conventions et d'incertitudes. Mais loin de vouer ces constructions savantes à une mise au rebut, nous leur assignons une place plus modeste que celle qui en ferait des prescriptions inattaquables : si les conventions sont acceptées par les acteurs et si ceux-ci accordent une certaine vraisemblance aux valeurs des variables et aux lois qui les relient, les résultats du modèle fournit une image de ce qui est souhaitable elle-même vraisemblable. Ici par exemple la réallocation fournie par l'ensemble formel PMSI/ENC/T2A. C'est en effet à ce type d'édifice que se consacrent les savants statisticiens, économistes, gestionnaires de l'ATIH (Agence Technique de l'Information Hospitalière), qui puisent dans leurs spécialités les ressources les plus sophistiquées pour perfectionner l'outil, notamment au niveau de la classification des séjours, qui en constitue la base essentielle. On a là un des « moteurs » de l'évolution continuelle du dispositif, et certainement sans fin prévisible, l'autre moteur venant de la société civile, sociétés savantes, associations de malades, établissements euxmêmes, exprimant des insatisfactions quant à la façon dont le modèle de financement prend en charge telle ou telle activité. Ces deux mécanismes se confortent l'un l'autre, mais comme on le voit, le concept de savoir/pouvoir foucaldien est insuffisant pour qualifier cette alliance, laissant dans l'ombre la frustration du technicien devant les imperfections de l'édifice, ainsi que les effets en retour des nouveaux savoirs générés par le jeu des forces en présence. Cela signifie que l'enrichissement du modèle et de ses apports de connaissances quant aux configurations possibles du futur suppose une réflexion collective sur ce qui est pertinent ou non aux yeux des uns ou des autres. Ces opérations de discussion et de correction existent dans le cas de la T2A, on l'a vu, mais posent question : le système de régulation a-t-il conscience de leur nécessité ? en prend-il acte ? les organiset-il ? qui y prend part et qui en est absent ?

- Une deuxième fonction d'apprentissage peut être conférée à l'instrumentation gestionnaire : non plus l'exploration du nouveau, mais une vision du présent, que là aussi l'esprit ordinaire, perdu dans une myriade de chiffres et l'accumulation de faits multiples et disparates ne saurait seul se construire. Il est incontestable à ce titre que le dispositif à la base de la T2A a contribué à lever quelque peu le voile d'opacité qui pesait depuis des décennies sur le système hospitalier. Une multitude de connaissances 
nouvelles, qu'il s'agisse des inégalités de ressources entre établissements, des activités connexes aux soins, comme l'enseignement et la recherche, de la variabilité de certains protocoles de soins, etc. ont été acquises par les uns et les autres. Dans le cadre du projet T2A lui- même, il s'agit au fond du savoir de pilotage du changement, permettant de repérer où l'on en est par rapport aux visées initiales et de fixer éventuellement des réorientations : qu'en est-il par exemple, des situations respectives des établissements au bout de sept années d'imposition du nouveau mode de financement? Là aussi de nombreuses mesures existent, mobilisées d'ailleurs souvent par des observateurs extérieurs à l'administration régulatrice (Inspection des Affaires Sociales, Cour des comptes etc.), souvent critiques, mais sur cette évaluation du présent se posent des questions analogues à celles que nous avons posées sur l'évaluation du futur : comment s'organise la réflexion sur des résultats, qui compte tenu de leur incomplétude et de leurs incertitudes devraient être soumis à une discussion systématique ? N'y a-t-il pas focalisation sur certains aspects, d'autres étant laissés dans l'ombre, comme la qualité et la sécurité, ou encore les conditions de travail du personnel hospitalier ? ${ }^{15}$

- La troisième fonction d'apprentissage liée à l'outil gestionnaire est moins connue, moins évidente. C'est celle qui émerge de l'analyse de son appropriation (ou non appropriation) par les acteurs qui sont censés l'intégrer dans leur activité productive. Une organisation est un champ de forces, orienté par les logiques professionnelles, les relations entre individus et groupes, mais aussi un ensemble de règles, de routines, d'instruments en place, et le nouvel outil de gestion ne va pas manquer de bousculer ce " déjà-là ", dont la force d'ancrage peut au contraire l'éliminer ou encore en dévoyer l'usage. Mais dans un même mouvement, les réactions, réticences, difficultés rencontrées par ceux qui portent l'outil révèlent la nature du champ de forces en présence et celle des problèmes structurels que pose l'insertion de la nouvelle logique gestionnaire. Cette fonction cognitive de l'outil a donné lieu à la constitution d'une méthodologie particulière en matière de recherche en gestion, fondée sur l'intervention ${ }^{16}$, et plus précisément sur ce que l'on peut appeler l'interaction instrumentée : un travail avec les parties prenantes d'un projet de changement pour l'outiller avec eux, les accompagner et évaluer, à partir des problèmes qu'ils expriment, les déterminants de leur action collective. C'est cette méthode qui a été appliquée dans les rares expériences visant à analyser les effets de la T2A dans les établissements, et qui a conduit à la constatation que ce mode de financement, malgré son objectif affiché de générer des outils de gestion internes et in fine une transformation des organisations, n'atteignait pas le cour de l'activité hospitalière, c'est-à-dire celle des processus de soin. Mais comme on l'a dit, ce type d'expérience a été très rare, le paradigme de la boîte noire étant suffisamment prégnant pour que le régulateur se prive d'observations consistantes sur la rencontre entre sa création instrumentale et les forces organisationnelles en présence.

On voit finalement que dans ces trois types d'apprentissage par l'instrument, l'ingrédient indispensable est constitué par l'interaction 
humaine, qu'il s'agisse de discussions sur les règles (savoir d'exploration), de réflexion collective sur la signification de telle ou telle mesure (savoir de pilotage), ou d'échanges sur la gouvernance des établissements (savoir organisationnel). C'est donc l'instauration d'un système particulier de relations qui à nos yeux est susceptible de s'opposer à ce qu'un dispositif instrumental se transforme en automatisme favorisant l'opportunisme des acteurs au détriment de l'apprentissage collectif, également on l'a vu des visées initiales de la réforme.

On ne veut pas dire que ces mêmes ingrédients sont complètement absents de la trajectoire de la T2A, mais ils interviennent de façon parcellaire, variable suivant les briques de l'édifice. Sur certains aspects, un processus organisé de concertation a été mis en place, sur beaucoup d'autres le changement s'opère par le biais de relations bilatérales, des négociations peu perceptibles par ceux qui ne sont pas impliqué, conduisant à des corrections consignées dans un dispositif se présentant chaque année sous la forme d'un arrêté tarifaire, comme fini, alors que chacun sait qu'il sera modifié l'année suivante et que l'interprétation des modifications enregistrées constitue un exercice impossible la plupart du temps. Plus précisément, si l'on reprend les trois sources d'apprentissage, ces discussions portant essentiellement sur les règles du modèle, restent pauvres sur les effets collatéraux (un comité d'évaluation de la T2A avait été mis en place à l'origine mais a été supprimé depuis) ; la faible appétence des établissements, d'ailleurs peu présents dans les procédures de concertation en place, à réorganiser les processus de soins rencontre de son côté peu d'échos du côté de l'Administration Centrale.

Un des inconvénients majeurs d'un tel système d'action est qu'il ne fait qu'alimenter la méfiance des uns et des autres, favoriser l'instauration d'une ère du soupçon, et accroître l'atmosphère de compétition, public versus privé, hôpitaux universitaires versus centres hospitaliers, spécialités versus toutes les autres etc. ; cela alors que les intentions malveillantes prêtées au régulateur ne résistent pas le plus souvent à une cohabitation de travail minimale avec des agents qui se révèlent pour l'essentiel livrés à un équilibrisme difficile entre les différents « outputs » qui surchargent le modèle.

Un principe alternatif de structuration de l'action collective autour et à partir de l'outil T2A serait de concevoir un dispositif organisé où instrumentation et apprentissages se construisent conjointement, avec mise en place de boucles de rétroaction alimentant des rendez-vous réguliers entre régulateurs et régulés. En d'autres termes l'instauration - ou la restauration - d'une évaluation continue, en même temps pilotage du système. Évaluation qui aille au-delà des statistiques actuelles et qui se préoccupe notamment des points aveugles du système en engageant des analyses nécessairement qualitatives.

Il s'agit sans doute là d'une vision quelque peu théorique, non seulement à cause de la complexité $\mathrm{du}$ dispositif technique et de la multitude de réglages à statuer, mais aussi parce que les cadres de l'Administration, tout en adoptant un mode 
de pensée nouveau, économique et gestionnaire, n'ont pas réellement changé la représentation qu'ils avaient de leur mode d'action sur la société civile ; il s'agit toujours d'élaborer des " solutions », qui seront « appliquées » à des assujettis aux réactions prévisibles, solutions traduites en lois, décrets, arrêtés ou circulaires. Ne s'est pas vraiment imposée l'idée que les comportements ne sont que partiellement prévisibles, surtout quand ils s'expriment dans des organisations qui les métabolisent, que les questions peuvent changer dans le processus lui-même, que les savoirs générés par ce dernier peuvent créer de nouvelles voies pour l'action, et que tous ces « accidents » constituent également des opportunités de nouveaux apprentissages.

Ce schéma, qui en quelque sorte fait passer d'une rationalité substantive à une rationalité procédurale, serait donc un pas considérable. Il consisterait aussi à ajouter aux figures de l'État, à la fois régalien, protecteur, planificateur, stratège, contractualiste - toutes ces figures que l'État a successivement épousées et qu'il a superposées - celle de " knowledge manager ». On en est loin lorsque l'on examine les aventures de la T2A, même si des germes d'une telle métamorphose peuvent s'y repérer. Pourtant cette philosophie de l'action organisée, qu'elle soit publique ou qu'elle concerne les entreprises, nous semble être la seule compatible avec la fragilité structurelle des outils qu'à présent elle manipule à loisir. Dans cette optique, l'instrumentation ne supprime pas la communication. Elles se transforment l'une l'autre dans la perspective de la construction d'un sens commun.

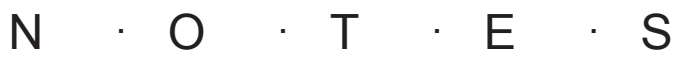

1. P. Lascoumes, P. Le Gales, Gouverner par les instruments, Paris, Sciences Po, 2004.

2. M. Berry, Une technologie invisible?, Paris, École polytechnique, 1983.

3. B. Jobert (dir.), Le tournant néolibéral en Europe, Paris, L'Harmattan, 1994.

4. B. Mas, F. Pierru, N. Smolski, R. Torrielli, L'hôpital en réanimation, Paris, Éditions du croquant, 2011.

5. J.C. Moisdon, «L'évaluation du changement organisationnel par l'approche de la rechercheintervention. L'exemple de la T2A », in Revue Française des Affaires Sociales, $n^{\circ}$ 64, Paris, juin 2010, pp. 213-226.

6. Z. Or, J. Bonastre, F. Journeau, C. Nestrigue, Activité, productivité, et qualité des soins des hôpitaux avant et après la T2A, Paris, Document de travail IRDES, 1993.

7. FHF, Évaluation de la T2A, Paris, Rapport, 2013.

8. H. Mintzberg, Structure et dynamique des organisations, Paris, Éditions d'Organisation, 1982.

9. C. Riveline, Cours d'évaluation des coûts, Paris, Mines Paris tech, 1995.

10. E. Minvielle, «Réconcilier standardisation et singularité : les enjeux de l'organisation de la prise en charge des malades ", in Ruptures, revue transdisciplinaire en santé, vol 7, n 1 , Paris, 2000, pp. 8-22.

11. Les pôles sont des regroupements de service, mis sous l'autorité d'un médecin, institutionnalisés par la loi de 2010 portant réforme des hôpitaux.

12. J.C. Moisdon, Le paradoxe de la boîte noire, in Droit et Société, n80, Paris, 2012, pp. 93-115.

13. A. Hatchuel, « Quel horizon pour les sciences de gestion? Vers une théorie de l'action collective", in Les Nouvelles fondations des sciences de gestion, 
Paris, Vuibert, 2000.

14. J.C. Moisdon, Du mode d'existence des outils de gestion, Paris, Seli Arslan, 1997.

15. F. Kletz, S. Damart., L. Brami, « Réformes de l'hôpital, crise à l'hôpital: une étude des liens entre réformes hospitalières et absentéisme des personnels soignants », in Politique et Management Public, n 29(3), Paris, pp. 541-561.

16. A. David, « La recherche-intervention, cadre général pour la recherche en management? », in Les Nouvelles fondations des sciences de gestion, Paris, Vuibert, 2000.

\section{$\mathrm{R} \cdot \mathrm{E} \cdot \mathrm{S} \cdot \mathrm{U} \cdot \mathrm{M} \cdot \mathrm{E}$}

L'article se propose d'analyser l'émergence et le développement d'un nouveau mode de financement des établissements de santé français : la tarification à l'activité (T2A). Largement inspirée de travaux microéconomiques anglo-saxons, la T2A repose sur un principe fort d'incitatif à l'efficience. Cet objectif rencontre en l'occurrence de sérieuses difficultés : d'une part le dispositif s'est progressivement complexifié, prenant en charge d'autres finalités et de multiples demandes de correction, et rendant le signal économique peu compréhensible, d'autre part les caractéristiques de l'organisation hospitalière se sont révélées peu favorables à une réorganisation des processus de soins. Cela ne veut pas dire que le bilan de la T2A est négatif (elle a d'autres effets divers difficiles à évaluer), mais sans doute qu'il convient de la modifier et de la piloter selon un processus davantage participatif prenant en compte ses fragilités structurelles.

\section{Abstract}

This article aims to analyse the emergence and the development of new funding arrangements for French hospitals: the casemix-based prospective payment system. Largely inspired by the Anglo-Saxon microeconomic theories, the new financing system is based on the principle of a strong incentive for efficiency. This goal encounters serious problems. On the one hand the system has gradually become much more complex, taking into account others purposes and numerous requests for changes, thus making the economic signal not easy to understand. On the other hand the hospital organization's characteristics turned out to be unfavorable to a reorganization of the care processes. This does not mean that the global conclusion is negative ; this payment system produces various effects which 
are difficult to assess. But there is probably a case for amending and piloting it in a more participatory process, taking into account its structural weaknesses. 
